\title{
Contour Boxplots: A Method for Characterizing Uncertainty in Feature Sets from Simulation Ensembles
}

\author{
Ross T. Whitaker, Senior Member, IEEE, Mahsa Mirzargar and Robert M. Kirby, Member, IEEE
}

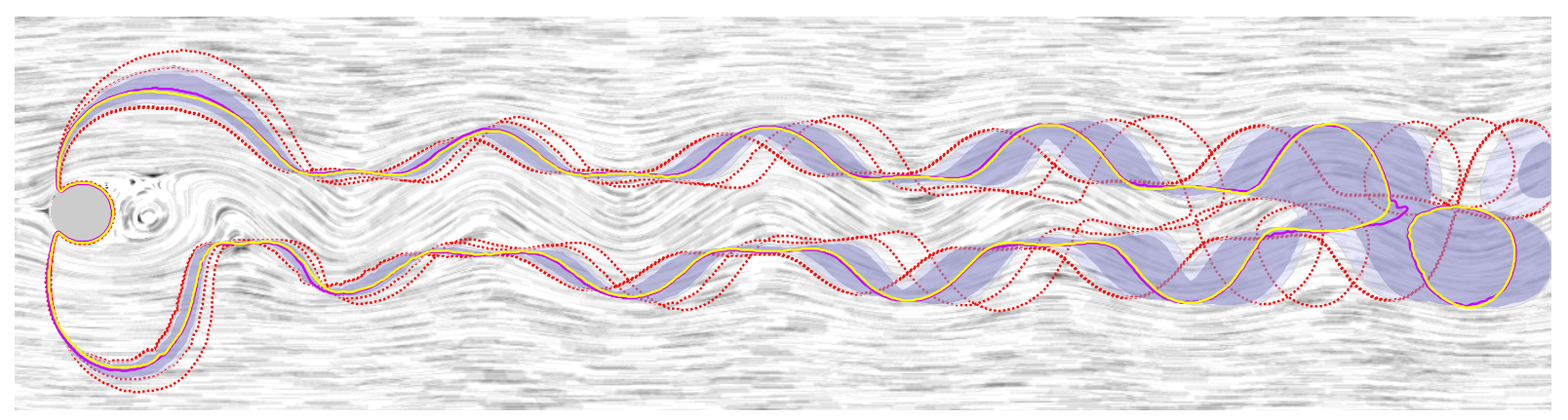

Fig. 1. Contour boxplot for an ensemble of the pressure field of a fluid flow simulation with a LIC background image for context.

\begin{abstract}
Ensembles of numerical simulations are used in a variety of applications, such as meteorology or computational solid mechanics, in order to quantify the uncertainty or possible error in a model or simulation. Deriving robust statistics and visualizing the variability of an ensemble is a challenging task and is usually accomplished through direct visualization of ensemble members or by providing aggregate representations such as an average or pointwise probabilities. In many cases, the interesting quantities in a simulation are not dense fields, but are sets of features that are often represented as thresholds on physical or derived quantities. In this paper, we introduce a generalization of boxplots, called contour boxplots, for visualization and exploration of ensembles of contours or level sets of functions. Conventional boxplots have been widely used as an exploratory or communicative tool for data analysis, and they typically show the median, mean, confidence intervals, and outliers of a population. The proposed contour boxplots are a generalization of functional boxplots, which build on the notion of data depth. Data depth approximates the extent to which a particular sample is centrally located within its density function. This produces a center-outward ordering that gives rise to the statistical quantities that are essential to boxplots. Here we present a generalization of functional data depth to contours and demonstrate methods for displaying the resulting boxplots for two-dimensional simulation data in weather forecasting and computational fluid dynamics.
\end{abstract}

Index Terms-Uncertainty visualization, Boxplots, band depth, ensemble visualization, order statistics.

\section{INTRODUCTION}

An important and widely used strategy within the area of uncertainty quantification (UQ) in simulation science is the construction of ensembles. Ensembles are collections of different simulations for the same physical phenomenon conducted with differing parameter values, different boundary or initial conditions, different instances of stochastic phenomena, different phenomenological models, different numerical regimes or parameters, or even different software systems. These ensembles are a means to represents sets of possible, likely, or reasonable outcomes from a simulation-based experiment or forecast. They can be used to study sensitivities to parameters, evaluate possible extremecase scenarios, and qualitatively or quantitatively assess the inherent variability or uncertainty in a simulation outcome. For instance, virtually all weather forecasting is based on an aggregation of forecasts using different simulation systems and different initial conditions [34]. Likewise, hurricane forecasting is typically done by considering outputs from different hurricane prediction software systems [5].

In analyzing the results of a simulation or a simulation ensemble, scientists are often interested in particular features of interest, rather than the dense fields of data we normally associate with physical simulations. In many cases these features can be represented as isocontours or level sets of the physical data or derived quantities. Some

Manuscript received 31 March 2013; accepted 1 August 2013; posted online 13 October 2013; mailed on 4 October 2013.

For information on obtaining reprints of this article, please send e-mail to:tvcg@computer.org. very important applications rely on ensembles of climate- or weatherderived features. For instance, thresholds on rainfall and temperature can affect the boundaries of agriculture regions. Air-quality warnings and corresponding emission restrictions are often related to tests or thresholds of temperature, wind, etc. In the forecasting of fire, risk experts rely on weather forecast ensembles and thresholds of temperature, wind speed, and relative humidity to establish warnings or alerts. Understanding how such threshold boundaries behave across an ensemble of forecasts or simulations is essential to assessing the uncertainty of these predictions/warnings and weighing that uncertainty against other costs and risk factors.

Ensembles are also used to study the behavior of physical phenomena. In fluid simulations, researchers are often interested in the behavior of eddies and turbulence to better understand the structure of regions that have low pressure or high vorticity. They use ensembles to establish the sensitivity or repeatability of their findings through time or across sets of parameters or models. In solid mechanics, engineers often want to understand which parts of an assembly undergo critical thresholds of force or deformation in order to predict patterns of failure or modify designs to address these potential weaknesses [38]. In computational biomechanics, simulations give rise to load patterns on joints, and loads beyond certain thresholds are a risk factor for excessive joint wear [11]. In all of these cases, ensembles can help to establish the variability in the boundaries of these critical regions and give simulation scientists some way of quantifying the confidence in their findings.

When considering the visualization of ensemble data there are a variety of different options. One very natural strategy is to compute statistics on the underlying fields of physical parameters [19]. Treating these fields or functions as vectors in linear spaces allows one 
to define distances, inner products, a corresponding statistical aggregates of mean, variance/covariance, etc. However, we will assert and demonstrate that statistical analyses of the underlying functions are not the same as analyses of the feature sets, which are isocontours of fields and derived quantities. Functions include modes of variability that are not important when considering particular isocontours. The notions of an average, median, or most representative sample have a different meaning (and result) when considering fields versus isocontours. Meanwhile, certain applications are particularly interested in the shapes, positions, and variability of the isocontours (contours) themselves, and thus statistical analyses and their corresponding visualizations must reflect this.

Because contours are not functions, but are instead manifolds or shapes, statistical analyses of ensembles of contours are particularly challenging, and there is an active field of research called statistical shape analysis, that seeks to establish the foundations that overcome these challenges [7]. Much of the work in shape analysis relies on assumptions about correspondence, topology, and alignment of shapes, and is difficult to apply in the contour setting without significantly more development. In this work we propose a somewhat simpler type of shape analysis that is motivated by a class of methods for statistical analysis of functions.

The development of this methodology is motivated by a set of criteria that we have established based on collaborations with domain scientists/engineers and the kinds of questions they are trying to answer with these ensembles.

Informative about contours/regions: As described above, visualizations should apply specifically to contours and convey statistical properties of their shapes, positions, etc.

Qualitative interpretation: Visualizations should provide high-level, qualitative interpretations of shapes of contours and variability.

Quantitative interpretation: Visualizations should display welldefined statistical content that allows for quantitative interpretation.

Statistical robustness: Aggregate quantities in visualizations should not be sensitive to a small number of examples in the ensemble that are significantly different from the others; such examples should be identified.

Aggregation preserving shape: Visualizations should display summary information but should not hide details that are characteristic of the typical properties of the contours.

This paper presents a new method for visualizing ensembles of contours. The method uses nonparametric statistical methods to quantify and display a set of information that is analogous to the mean, median, order statistics, and outliers that are typical in conventional boxplots. Thus, we call the method contour boxplots. Contour boxplots rely on the quantification of statistical depth-how central or deep an instance is within a distribution. While there are many ways to characterize depth, for this work we present a novel method for computing depth of contours, for which there is no depth measure to date. The proposed contour depth is a generalization of the method of band depth, which has been presented in the statistics literature to quantify and visualize ensembles of functions [17]. Band depth exhibits some sensitivity to the shapes of functions (rather than just their position), which is important in this context. The proposed generalization of band depth is proven to be mathematically equivalent to functional band depth under specific conditions, but also much more general. We also show how this method of band depth can be used to construct a set of visual primitives (lines and polygons) that display the information one would normally associate with a boxplot. Thus, this new analysis and visualization technology offers a statistically sound and visually effective way of presenting ensemble results for feature sets. We evaluate and analyze the proposed method and some other state-of-the-art approaches in relation to the list of criteria above.

The rest of the paper proceeds as follows. The next section gives an overview of related work and characterizes the proposed contour boxplot method in that context. Section 3 briefly reviews the technical background for band depth and functional boxplots, describes the proposed generalization, and establishes equivalences for special classes of inputs. Section 4 describes the implementation and the visualization strategies for contour boxplots. Section 5 verifies the behavior of the method on controlled experiments and then shows results for two different applications - computational fluid dynamics and weather forecasting. We summarize our findings and conclude in Section 6.

\section{BACKgROUND ANd ReLATEd Work}

The origins of the recent research activity in "uncertainty visualization" are not entirely clear; however, most would acknowledge that the early works of Pang et al. (e.g., [20, 16, 19]) combined with Johnson and Sanderson's call for "visualization error bar" [12] signaled an upturn in interest for methods designed to represent the output of the process of uncertainty quantification in experiments and simulation. A salient review and taxonomy of the state-of-the-art in uncertainty visualization is given in [29]. We review here those works most relevant to the motivation and contributions of our current work: those related to the visualization of (simulation) data-derived features extracted from fields having uncertainty information in the form of distributions (represented in terms of a model of the distribution or as an ensemble).

Grigoryan and Rheingans [9] introduced point-based primitives to show surface uncertainty by displacing individual surface points along its normal by an amount proportional to a random number multiplied with the local uncertainty value, hence creating a "fuzzy surface" to convey the surface uncertainty. Texture and/or color are employed to denote uncertainty through a modification of a direct volume rendering technique in [6]. Other proposed methods to visualize surface uncertainty include fat surfaces [19], likelihood and confidence maps [21], and point primitives for rendering uncertain isosurfaces [10]. Following from this idea, Kniss et al. [15] developed a volume visualization method that incorporates the fuzzy classification into the visualization cycle to allow users to interactively explore the uncertainty, risk, and probabilistic decision of surface boundaries. Rhodes et al. [31] proposed an isosurface rendering technique for multiresolution volumetric data while incorporating the error present in the data. In summary, all such previous attempts at visualizing the uncertainty of extracted features fail to meet one or more of our aforementioned motivating criteria: in particular, they do not maintain both a qualitative and quantitative means of interpretation of the certainty, and it is not clear that they make any attempt to robustly summarize the set of shapes in an ensemble, but mostly seem to convey magnitudes in potential error or variability in point positions.

In recent years, there has been a move within the community to incorporate statistical models directly into the process of detecting isocontours. The concept of Level Crossing Probability (LCP) was introduced to quantify and visualize uncertain scalar fields [26], where they introduced a model for uncertain spatial data and the corresponding spatial distribution of uncertain isocontours. As an extension of this method, a probabilistic version of the marching cubes algorithm was proposed in [28], where the authors take into account scalar fields in which the data points are possibly correlated. Pfaffelmoser et al. [23] proposed the isosurface-first-crossing-probability (IFCP) algorithm as an efficient way to calculate the probability incrementally along a ray cast through a correlated random variable field. In [27], the authors introduced an approximation to calculate cell-wise level-crossing probabilities for uncertain scalar fields as a preprocessing stage. With a discretized Gaussian assumption, they have designed a tool to explore the LCP interactively. The LCP methodology does provide quantitative estimation error bounds. However, the visualization of the LCP (even when accounting for local correlations) is not able capture the global statistics of the field that are often the most important aspect of understanding the ensemble of simulations and their associated physics. Furthermore, while the method is quantitative by construction, it provides only a qualitative visualization of the variability, and thus does not satisfy the criteria we have established in the Introduction. This will be demonstrated on specific examples later in this paper.

A related work [24], proposed a technique to represent the global 
correlation structure of 2D discrete scalar fields through the introduction of a correlation strength model for discrete points. While their correlation model can be defined over the whole domain, to make it practical, a clustering approach was introduced, and correlation values are only calculated locally. That work was extended in [22] for probabilistic local feature extraction for crisp vector fields considering a local correlation structure on the uncertain vector field. The proposed framework was used to find probabilities of singularities (e.g., sink or saddle point) using the local neighborhoods. Although that method does give a quantitative interpretation of the variability of features, the necessary reduction in degrees of freedom through local approximations limits its ability to convey a sense of the typical shape of members drawn from the distribution. Furthermore, the reliance on parametric models presents challenge in the case of non-normal distributions and outliers.

An alternative approach to tackling the quantification issue is to attempt to visualize the ensemble data directly and let the user interpret the underlying variability and uncertainty qualitatively. Joseph et al. [14] introduce a visualization system for displaying uncertainty in isosurfaces and facilitating isosurface comparison. Phadke et al. [25] proposed scaled data driven spheres (SDDS) and attribute blocks to visualize single (or multiple) ensemble members. One can also use descriptive statistics of the ensemble data to simplify the interpretation. Ensemble-Vis [30] was introduced as a flexible infrastructure to convey statistical properties of ensemble data along with multiple views of various fields of interest to enhance visual analysis of ensemble data. In addition to these general approaches, there have also been domainfocused attempts at direct ensemble visualization. Noodles [33] is an example of an interactive visualization tool that has been designed for visualizing weather forecast data where the uncertainty has been quantified for each of the ensemble members independently from the ensemble mean. In [5], hurricane track predictions are examined in an similar manner, and an ensemble visualization technique is proposed as an alternative to the "error cone" visualization typically displayed to the public in hurricane warnings. These methodologies, however, fail to provide quantitative summaries or aggregates, and place a larger cognitive burden on the user to interpret the statistical meaning of the many examples. That is, they inadequately summarize shape in a way that readily leads to a precise, quantitative interpretation.

\section{Band Depth Method and Its Generalization to Con- TOURS}

Here we begin with a review of the concept of statistical depth and the definition of band depth, which is a particular depth measure that is sensitive to the shapes and heights of functions.

Order statistics (e.g., percentiles, median) require an ordering on the data. For scalar data, this ordering is obtained by simply sorting the values. Bagplots proposed in [32] contained a generalization of ranks for bivariate points, which relies on the notion of halfspace depth [37]. A bagplot consists of a bag denoting $50 \%$ of the data and a fence (inflation of the bag by a factor of three) to distinguish the outliers. For multidimensional data, the concept of ordering may be more complicated, especially when one wants to capture specific properties, such as geometric relationships between variables. Of course, one could order each coordinate (or degree of freedom) independently of the others, but such an approach essentially assumes independence, and ignores structure in the data that is reflected in the interdependency (e.g., correlation) of coordinates.

Statisticians have developed a concept of data depth for nonparametric, multivariate data analysis. Data depth provides center-outward orderings of multivariate data and has led to a variety of new nonparametric multivariate statistical analyses, i.e., methods not reliant upon assumptions about the underlying distributions. Data depth quantifies how central (or deep) a particular sample is within a given cloud of data. The deeper samples are considered more representative of the data as a whole, and the shallowest samples can be considered outliers. Thus, depth allows one to perform certain kinds of order statistics on multidimensional data in a way that is general but does not ignore statistical relationships between coordinates.
The statistics literature describes a variety of different methods for data depth, but a recent development, called band depth [18], is particularly relevant for the goals of this paper. Band depth is defined on an ensemble of functions, $f_{i}: \mathscr{D} \mapsto \mathscr{R} i=1, \ldots n$. For this discussion, $\mathscr{D}$ and $\mathscr{R}$ are intervals in $\Re$. The band depth of each function is the probability that the function lies within the band defined by a random selection of $j$ functions from the distribution. For instance, a function $g(x)$ that lies in the band of $j$ randomly selected functions $f_{1}(x), \ldots, f_{j}(x)$ satisfies the following:

$$
\begin{array}{r}
g(x) \subset B\left(f_{1}(x), \ldots f_{j}(x)\right) \text { iff } \\
\forall x \min _{k=1, \ldots, j} f_{k}(x) \leq g(x) \leq \max _{k=1, \ldots, j} f_{k}(x) .
\end{array}
$$

Fig. 2a shows the construction of this band graphically for $j=3$. We can see that a function lies in the band defined by $j$ other functions if it lies between the upper and lower envelopes formed by those functions. The band depth for a given $j$ is the probability that a function falls into the band formed by an arbitrary set of $j$ other functions chosen at random from the probability distribution associated with the ensemble:

$$
\mathrm{BD}^{j}(g(x))=P\left[g(x) \subset \mathrm{B}\left(f_{1}(x), \ldots f_{j}(x)\right)\right],
$$

where the indices enumerate members of a set in no particular order. The notion of band depth has been found to be more robust if one considers the sum of all smaller sets to form the band. That is

$$
\mathrm{BD}_{J}(g(x))=\sum_{j=2}^{J} \mathrm{BD}^{j}(g(x)) .
$$

The probability $P[\cdot]$ is computed by a sample mean of the indicator function associated with Eq. 2, which is formed by evaluating BD over all appropriately sized subsets from the given ensemble (excluding $g$ ). The size of subsets should be chosen to prevent degenerate cases where no bands are sufficiently wide to contain the function or too many examples have the same depth. For instance, if one considers $j=3$ for an ensemble containing 10 functions, a particular example would be tested against $C(9,3)=84$ bands in order to determine the probability in Eq. 2.

The band depth is computed for each member of the ensemble and can be used, as described in [35, 36], to visualize summary statistics for an ensemble of functions, as shown in Fig. 2b-c. A function boxplot, therefore, shows the median (sample with the greatest depth-maximum BD), bands associated with percentiles (e.g., band containing $50 \%$ of the examples) and outliers (e.g., samples $f$ with $B D(f)>1.5 \times B D\left(f_{50 \%}\right)$, where $f_{50 \%}$ is the function that is at the middle of the sorted list of BD values from the ensemble. Notice that this definition of data depth allows one to capture shape differences in functions. That is, functions that are near the center of the distribution but have irregular shapes will have a lower probability of falling within a particular band of randomly chosen samples. This method also captures relationships between values across the domain, which may be lost if one uses conventional boxplots to describe pointwise statistics of the ensemble (as shown Fig. 2c).

Theoretical results on this definition of band depth show that it satisfies several important statistical criteria. The sample mean of the probability of falling into a particular band size converges to the expectation as the size of the ensemble goes to infinity. For symmetric distributions, the sample with maximum depth converges to the center of the distribution. Likewise, samples that are infinitely far from that center will have band depths that approach zero. Finally, for the case of univariate data band depth is equivalent to conventional order statistics.

In practice, with too few samples and too much variability in shape, there are simply not enough subsets, and many samples can have a BD that is very low or zero, which can interfere with the ability to discriminate the depths of different samples, and therefore [17] propose a modified band depth (MBD), which computes the expected value of the amount of the domain $\mathscr{D}$ for which a function $g(x)$ lies in the band. 


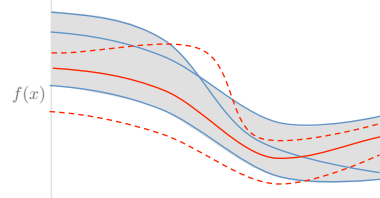

(a)

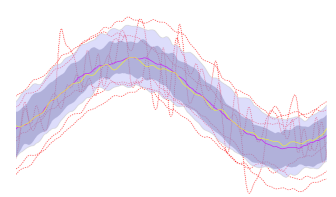

(c)

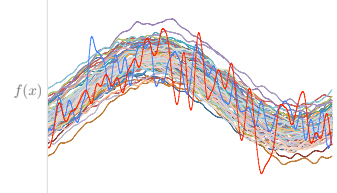

(b)

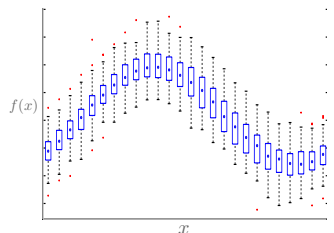

(d)
Fig. 2. Examples of function band depth and function boxplots. (a) For band depth, three different curves (in blue) form a band (in grey) against which three other (red) curves are tested, with only the solid curve falling in the band. (b) A set of 80 simulated, noisy curves with some outliers in shape and position. (c) A version of the function boxplot, as proposed by $[35,36]$. (d) A conventional, pointwise, boxplot loses information about shapes of curves in the ensemble.

This modification produces more reliable results in high variability situations, but significantly reduces the sensitivity of the method to the shapes of functions, because irregularly shaped functions few bands that contain them perfectly but many bands that contain them approximately.

For the analysis of contours, we will generalize this definition of band depth and propose an alternative modification for dealing with scenarios with relatively small sample size and high variability. We begin with a new definition of band depth, which operates on sets, and show that this definition is equivalent to function band depth for the special case of functions.

Consider an ensemble of sets $E=\left\{S_{1}, \ldots, S_{n}\right\}$, where $S_{i} \subset U$, and $U$ is the universal set. We say that a set $S \in E$ is an element of the band of a collection of $j$ other sets $S_{1}, \ldots, S_{j} \in E$ if it is bounded by their union and intersection. That is,

$$
S \in \operatorname{sB}\left(S_{1}, \ldots S_{j}\right) \Longleftrightarrow \bigcap_{k=1}^{j} S_{k} \subset S \subset \bigcup_{k=1}^{j} S_{k} .
$$

From this we can define the set band depth (sBD) as the probability that a set lies in the band of a random selection of sets from the ensemble:

$$
\operatorname{sBD}_{J}(S)=\sum_{j=2}^{J} P\left[S \in \mathrm{sB}\left(S_{1}, \ldots S_{j}\right)\right] .
$$

As with the function $\mathrm{BD}, \mathrm{sBD}_{J}$ is computed by taking all appropriately sized subsets of $E$.

To apply sBD to isocontours, we will consider the subsets in the plane enclosed by those contours, as in Fig. 3a. Thus, given a set of fields $F_{1}(x, y), \ldots, F_{n}(x, y)$ the algorithm for computing the $\mathrm{sBD}$ (for a particular value of $j$ ) of isocontours with value $q$ is as follows:

1. Compute the sets (represented as binary functions on a grid): $S_{j}=\{(x, y) \mid F(x, y)>q\}$ for $i=1, \ldots n$.

2. For $i=1$ to $n$

(a) Initialize $P_{i}=0$

i. For each subset of $\left\{S_{1}, \ldots, S_{n}\right\}$ of size $j$ and not containing $S_{i}$ (call it $Q$ )

A. Compute $S_{U}=\bigcup_{S_{k} \in Q} S_{k}$ and $S_{I}=\bigcap_{S_{k} \in Q} S_{k}$ (using min and max operations on the grid)

B. If $S_{I} \subset S_{i} \subset S_{U}$ (testing using differences on the grid), increment $P_{i}$

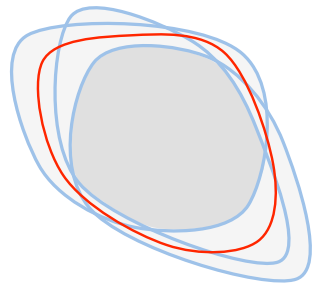

(a)

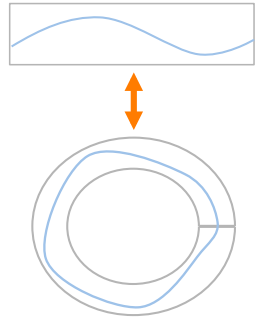

(b)
Fig. 3. (a) For three test contours (blue), a red contour lies in the contour band if it encloses the intersections of the regions (grey) and within the union of those regions (light grey). (b) For topologies in the plane that map to the graph of a function, set band depth in the plane is exactly equivalent to function band depth.

ii. Normalize $P_{i}$ by dividing by the number of subsets $((n-1)$-choose- $r)$

\section{Sort the values of $P_{i}$.}

Visualizations are constructed from the depth-sorted contours, as described in the next section.

We call the application of set BD to isocontours contour band depth (cBD). Of course, nothing in the formulation limits this method to 2D domains. Indeed, in the results section we will show results for time varying 2D fields, which will be treated as 3D fields, but we show them as a 2D sequence in time. In this paper we consider the display of contour boxplots only in 2D-the very challenging problem of visualizing multiple, nested 3D surfaces is beyond the scope of this paper.

The proposed formulation of set band depth (sBD) and its application to contours (cBD) exhibit a set of theoretical properties that are important in understanding its behavior and potential applications. These properties are described, with sketches of proofs, in the Appendix. First, the function BD method of [17] is a special case of $\mathrm{sBD}$, where the sets are given by regions in the plane. Thus, sBD is a more general and broadly applicable version of band depth. Second, while contour band depth (cBD) applies to contours or level sets generally, it is equivalent to function BD in the very special case where the contours can be represented as functions. Thus, cBD is also a generalization of function BD. Therefore, one of the contributions of this work is our introduction of more general definitions of band depth.

This raises important questions about $\mathrm{cBD}$ and what properties it exhibits with respect to contours. Here we identify two properties of interest, both of which are described more rigorously in the Appendix. First, even though cBD operates with respect to the sets associated with interiors $(F(x, y)>q)$ of contours, it is invariant with respect to the definition of interior/exterior. Thus, we can say that cBD analysis is a property of the set of contours themselves rather than the functions from which they are generated. Second, the band requirement given by Eq. 4 is consistent with the conditions associated with level sets in a contour tree [4]. This is important because it means that we can use many of the results regarding the topological structure of contour trees to better understand $\mathrm{cBD}$. Also, this suggests that the proposed formulation may open possibilities for nonparametric statistical analyses of contour trees.

As with function $\mathrm{BD}, \mathrm{cBD}$ will produce unsatisfactory results $(e . g$, many contours may have $P=0$ ) if the ensemble is relatively small and the contours vary significantly in shape. The modified band depth approach [17] to alleviate this problem, which uses an integral of the dependent variable for values within the band, is not immediately applicable to this context. The contour approach has no independent variable, and thus integrals would need to be defined on contour length in a way that accommodates different topologies. Instead, we take an alternative approach that generalizes our set definition of band depth. The strategy is to relax the definition of subset to allow a small percentage of each set to violate the classical definition. Thus we define an epsilon subset: 


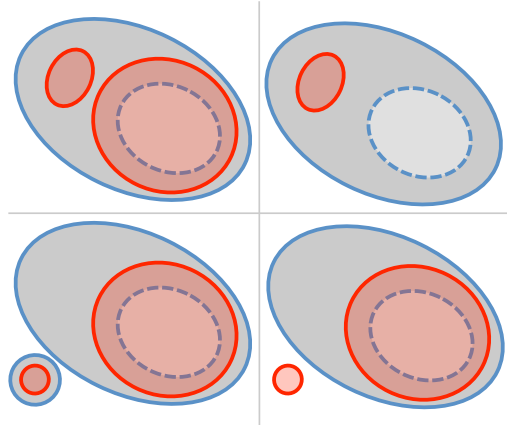

Fig. 4. Examples of how the contour band depth deals with topological differences. A contour (red) is tested against the contour of a union (solid) and an intersection (dashed blue). Left: two examples of different topologies satisfy the contour band property and represent properly nested contour trees of some continuous function. Right: two examples do not satisfy the contour band property and do not represent an ordering consistent with any continuous function.

$$
A \subset_{\varepsilon} B \Longleftrightarrow|A|=0 \text { or } \frac{|A-B|}{|A|}<\varepsilon,
$$

and the epsilon set band is

$$
S \in \mathrm{sB}_{\varepsilon}\left(S_{1}, \ldots S_{j}\right) \Longleftrightarrow \bigcap_{k=1}^{j} S_{k} \subset_{\varepsilon} S \subset_{\varepsilon} \bigcup_{k=1}^{j} S_{k} .
$$

Likewise, $\mathrm{sBD}_{\varepsilon}$ is defined from $\mathrm{sB}_{\varepsilon}$. This allows a softer definition of the band while retaining a sensitivity to contour shape, especially for very small $\varepsilon$. This does introduce the free parameter $\varepsilon$, but, as described in the next section, this can be determined automatically by examining the results of the subset operations across the ensemble and choosing $\varepsilon$ to ensure some simple, general criteria.

\section{Methods}

Here we discuss several important aspects of the computation of contour band depth for elements of an ensemble and the construction of visualizations. For computation of cBD, we use $J=2$ throughout this paper. As noted in the literature on function $\mathrm{BD}$, the smaller values of $J$ allow greater sensitivity to shape (two functions/contours of very different shapes tend to cross and violate the band condition). Smaller $J$ values are significantly faster to compute. The tendency for ties in the $J=2$ case is best alleviated by using the modified definition of band depth, $\mathrm{sBD}_{\varepsilon}$ for contours.

The use of $\mathrm{sBD}_{\varepsilon}$ for contours introduces a free parameter $\varepsilon$, which must be set or tuned for a particular ensemble or visualization. While some situations might benefit from exposing this parameter to users, for this work we have developed a set of rules to define this parameter automatically, incorporating some high level knowledge about the structure of the distribution. Here we present one of many possible ways in which $\varepsilon$ can be tuned automatically in order to produce the most informative ordering of the data.

The proposed method is to use the loops over different $J=2$ subsets to compute, instead of a binary variable, the percentage of mismatch between different set operations, $|A-B| /|A|$ in Eq.6 (take the worst/max of the union and intersection operations for a given comparison). If there are $m$ sets of $J=2$ against which to compare $n$ contours, this gives an $m \times n$ matrix, which we denote as $m_{i j}$. If we threshold for a particular choice of $\varepsilon$, the sum along column (divided by $m$ ) gives $\mathrm{cBD}_{\varepsilon}$ for that row/sample. We denote an element-wise threshold operation for the matrix as $T_{\mathcal{E}}\left(m_{i j}\right)$. If we sum across the rows and columns of $T_{\mathcal{E}}\left(m_{i j}\right)$ and normalize, we get the sample mean of the band depth for a particular choice of $\varepsilon$.

Here we consider a simple model. A one-dimensional probability density, $p(x)$, has cumulative distribution $c(x)$, so that $c^{\prime}(x)=p(x)$. For $J=2$, a particular sample $x$ falls in the band of (i.e., between) two randomly chosen samples if one sample is less than $x$ and the other sample is greater than $x$. Those probabilities are given by the cumulative distribution (and its complement). Thus the expected value for the band depth is:

$$
\begin{aligned}
E_{\mathrm{BD}} & =\int_{-\infty}^{\infty}(1-c(x)) c(x) p(x) d x \\
& =\int_{-\infty}^{\infty}(1-c(x)) c(x) c^{\prime}(x) d x=\frac{1}{6} .
\end{aligned}
$$

Consequently, the expected value of the band depth is an invariant, and for any one-dimensional probability distribution it is $1 / 6$. We do not know the dimensionality of the underlying distribution of a set of contours. However, as a rule of thumb, we can say that the most generous choice of $\varepsilon$ is the one associated with a 1D distribution of the data. To find that threshold on the soft subset, we can perform a binary search on $\varepsilon$ using the minimum values along the columns of matrix $m_{i j}$ to satisfy:

$$
\frac{1}{m n} \sum_{i=1}^{n} \sum_{j=1}^{m} T_{\varepsilon}\left(m_{i j}\right)=\frac{1}{6} .
$$

We have found this automatic method for choosing $\varepsilon$ to produce consistently useful band depth values, and have used this method for all of the results in this paper.

As part of the visualization, we also identify outliers. From the statistics literature, there is no precise, universal definition of an outlier. Some particular methods apply if one assumes a particular distribution (e.g., normal), which is not applicable to the nonparametric approach we are taking here. Outliers are typically samples that are rare. For this work, in order to capture rarity, we will define outliers as samples that have zero band depth-that is, the samples that cannot be bound (via contour bands) by any other pair of samples.

For some examples in this paper, we will perform the $\mathrm{cBD}$ analysis in space and time. For this, the definitions (and theoretical results) extend quite readily. We can compute set differences and sizes on regions in 2D and sum across time in order to perform soft thresholds. This analysis gives us the band depth of a full time-space surface associated with a threshold value.

All of the visualizations in this paper are of 2D domains, although some are dynamic. Our strategy is to show a set of information resembling that of a conventional boxplot of scalar data. Thus, we trim the data, removing outliers, which are shown separately as thin, dashed, red curves. We show bands associated with $50 \%$ and $100 \%$ data depths. These bands are easily computed as the difference of the union of a set of regions minus their intersection (as in the cBD definition). For display we render these bands as solid, but transparent, relatively neutral colors so that a user can see data underneath these bands to better understand their overall position. We render the contour with the greatest depth, which is the median, or the sample that is most representative of the ensemble as a solid, yellow line. We compute the mean of the ensemble as the contour associated with the region contained in greater than $50 \%$ of the samples in the ensemble, and render that as a purple line. Results of these rendering choices are shown in the next section.

\section{Results and Applications}

In this section, we discuss the experimental results using contour boxplots based on cBD to address the challenge of visualizing and interpreting the variability in ensemble data using several experimental examples. We also show comparisons against relatively simple operations on fields and another state-of-the-art method for quantifying error in isocontours. We begin with a synthetic example and then show two examples from simulation science applications.

The synthetic ensemble consists of 80 members, each of which is an isocontours of the implicit formulation of an ellipse with different size, rotation, and ellipticity. Furthermore, the shapes have been contaminated with correlated random noise. We have added several outliers in size as well as two examples where the shapes are generally very close to the mean but have been corrupted by relatively large amounts of correlated noise to simulate shape outliers. Figure 5 shows the collection of contours associated with the zero level sets of these implicit 


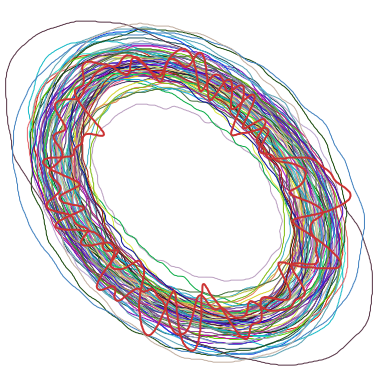

(a)

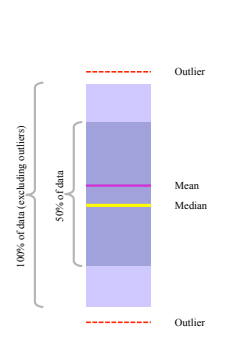

(b)

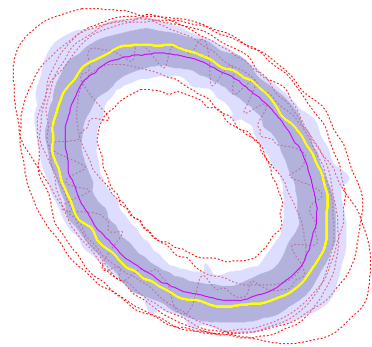

(c)

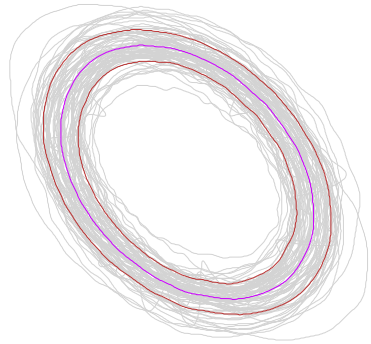

(d)

Fig. 5. (a) Synthetic ensemble data including isocontours of 80 randomly contaminated implicit ellipses formulation. (b) A legend for the display used in the contour boxplot. (c) Contour boxplot for the synthetic dataset. (d) For comparison, the isocontours of the mean of 1 standard deviation of the implicit functions.

functions. Because the topologies of these examples are constrained, (as illustrated in Fig. 3b), we know that this example is equivalent to the function boxplot example in Fig. 2. The soft subset threshold, $\varepsilon$, is chosen automatically using Eq. 9, and we list the values of $\varepsilon$ for all of our results in this section in Table 1. In these cases, the practical affects of choosing $\varepsilon$ are that if we choose $\varepsilon=0$ (full subset relationship), there is a significant fraction of the ensemble members that have no containing bands $\mathrm{cBD}_{\mathrm{j}}=0$, and they are all tied for the lowest data depth. If $\varepsilon$ is set significantly larger than zero, then we lose the sensitivity to shape, and, in the very extreme case (e.g., $\varepsilon \approx 1)$, many examples may be tied for very high depth.

Table 1. The soft subset thresholds

\begin{tabular}{cc} 
Experimental Example & Soft Subset Threshold $(\varepsilon)$ \\
\hline Synthetic Ensemble & 0.0001 \\
Fluid Simulation Ensemble & 0.005 \\
Fluid Region of Interest Ensemble & 0.001 \\
Weather Forecast Ensemble & 0.009
\end{tabular}

Figure $5 \mathrm{c}$ illustrates the sensitivity of the $\mathrm{cBD}$, even with the soft subset concept given by Eq. 7. This example shows that using cBD to construct contour boxplots will provide both qualitative and quantitative information about the variability among ensemble members using the notion of $50 \%$ percentile band. Moreover, the contour boxplot identifies and displays both the shape and the positional outliers. Notice that the median (shown as a yellow curve) is not centered in the $50 \%$ band, whereas the mean (shown as a magenta curve) is. This is expected, because the median in these examples is a representative contour from the ensemble, and there are no contours that are perfectly centered within the band. This is especially true in the context of the multidimensional variability (in size, shape, orientation, and noise) that we used to generate these examples. Likewise, the mean shows a smoothness that is not typical of the population. Thus, both contours may be useful in this context.

One of the benchmark methods against which we compare is extraction of the isocontour from the averaged field data (implicit functions), with bounding contours based on the standard deviation at each pixel. This simple approach is indicative of a broad class of methods for quantifying uncertainty in contours (e.g., [15]), which model uncertainty as the local sensitivity of the contour/surface to variations in the function or isovalue. Figure $5 \mathrm{~d}$ shows the isocontours of the mean and \pm 1 standard deviation of our synthetic dataset. Using the sample mean of the field removes (smoothes out) the details of the shape of individual ensemble members. Furthermore, while outlier detection on the fields is, in principle, possible, shape outliers are necessarily unusual with respect to their set of field values.

Our second set of experimental results is motivated by computational fluid dynamics applications in which scientists are interested in studying the dynamics of the fluid fields and the variability of features they observe in the flow. As fluid passes a solid object, eddies will periodically form and move along the eddy lines (i.e., the vortex street) that form behind the object. Simulations are often compared against experiments in controlled conditions. In practice, however, there are usually

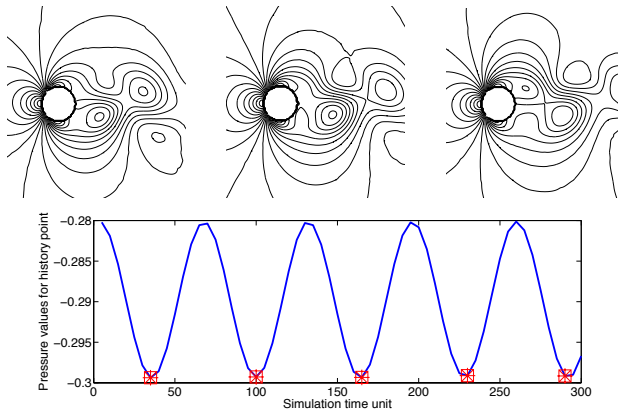

Fig. 6. Top: Contours of a pressure field for three consecutive time points show that the obstacle sheds eddies on a cyclical pattern. Bottom: Pressure values over simulation time for a single point allow one to snapshot a consistent times in the cycle.

small deviations in parameters, such as boundary velocities and temperature. For instance, the viscosity of fluid changes with temperature, which affects the Reynolds number of the simulation-an important parameter in vortex formation. Here we use contour boxplots to visualize the variability in shapes of the eddies among simulations with different parameter settings. For this example, we study the scenario of a 2D fluid moving past a solid disc, which generates a wake and series of eddies (top and bottom) that move in a cyclical pattern down the edges of the wake (eddy lines).

For the simulations, we use the 2D incompressible Navier-Stokes solver as part of the Nektar ++ software package [2] to generate an ensemble with 40 members, where each ensemble member is a simulation with Reynolds number and inlet velocity chosen randomly. The inlet velocity values are randomly drawn from a normal distribution with a mean value of 1 and standard deviation of \pm 0.01 (in non-dimensionalized units); likewise, Reynolds numbers are generated from a normal distribution with mean value of 130 and standard deviation of \pm 3 .

The simulations are of course dynamic, and the time courses and eddy formation patterns of each one are slightly different. However, because eddy formation is cyclical, we can compare simulations using snapshots for each simulation corresponding to approximately the same point in the eddy formation cycle. This is done as follows. After running the simulation long enough that the flow is well-developed (i.e., beyond the transient stage), we pick a particular point, directly behind the disc/obstacle, to observe the pressure values over time. The center of an eddy is typically a minimum in pressure, and thus as the eddies pass the history point, their pressure footprint through time will have a very regular periodic behavior, as in Fig. 6, where the red squares, at the pressure minima indicate the ensemble snapshot. Thus, we can capture synchronized samples from a dynamic simulation by timing them with this cycle, and all ensemble members are at approximately the same phase in the vortex shedding process. We also normalize the pressure field of each simulation using the averaged pressure value (over time) of the history point taken in the wake of the cylinder. Isovalues are extracted as a fixed level set value relative to 
(a)

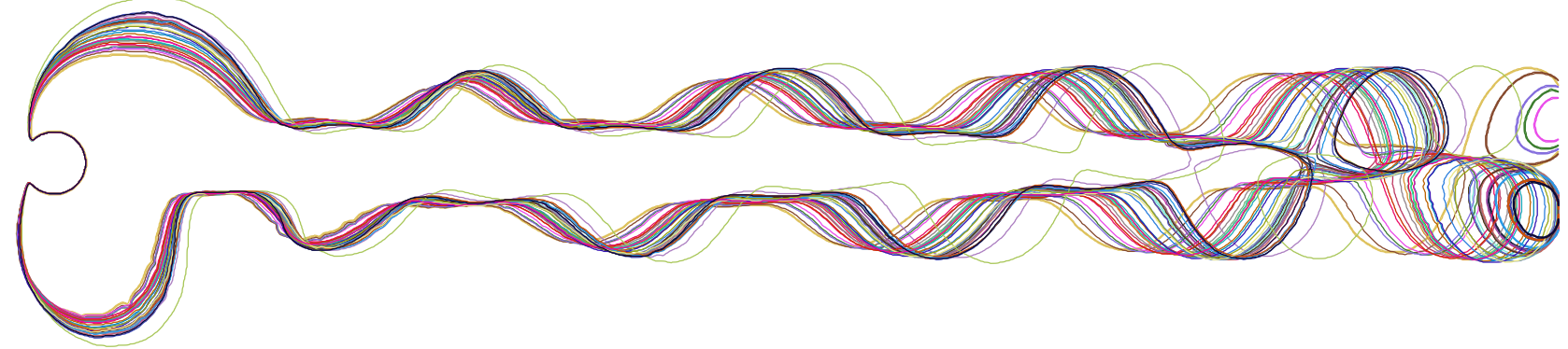

(b)

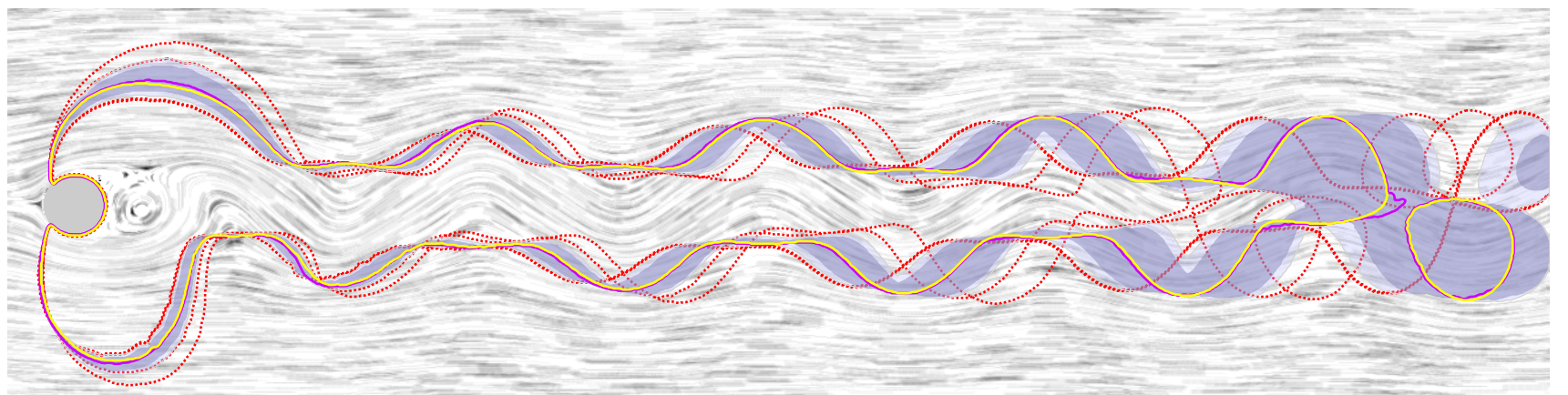

(c)

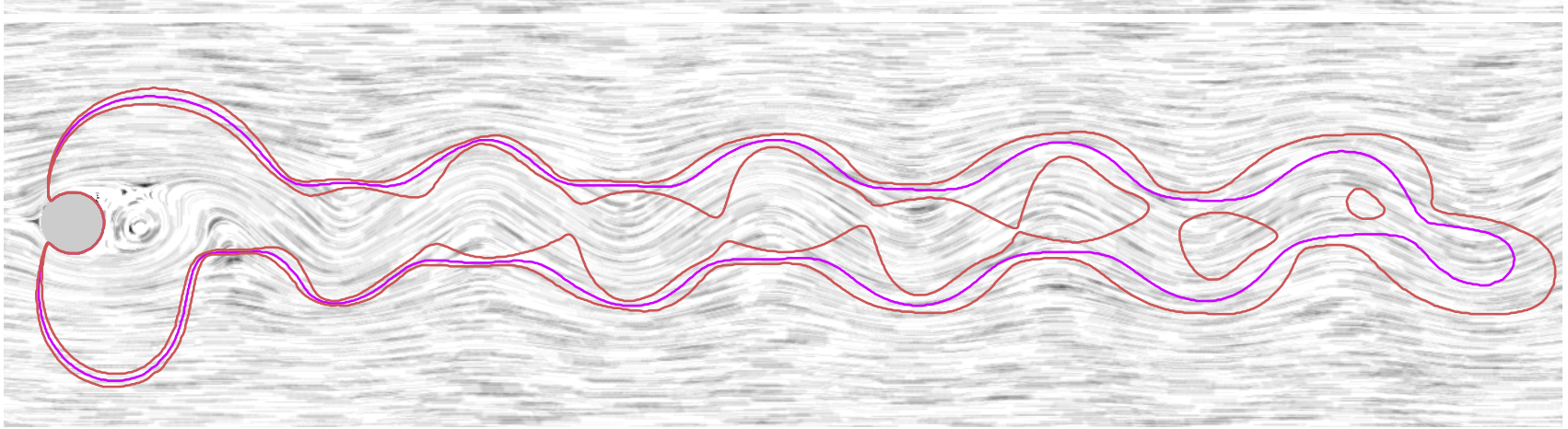

(d)

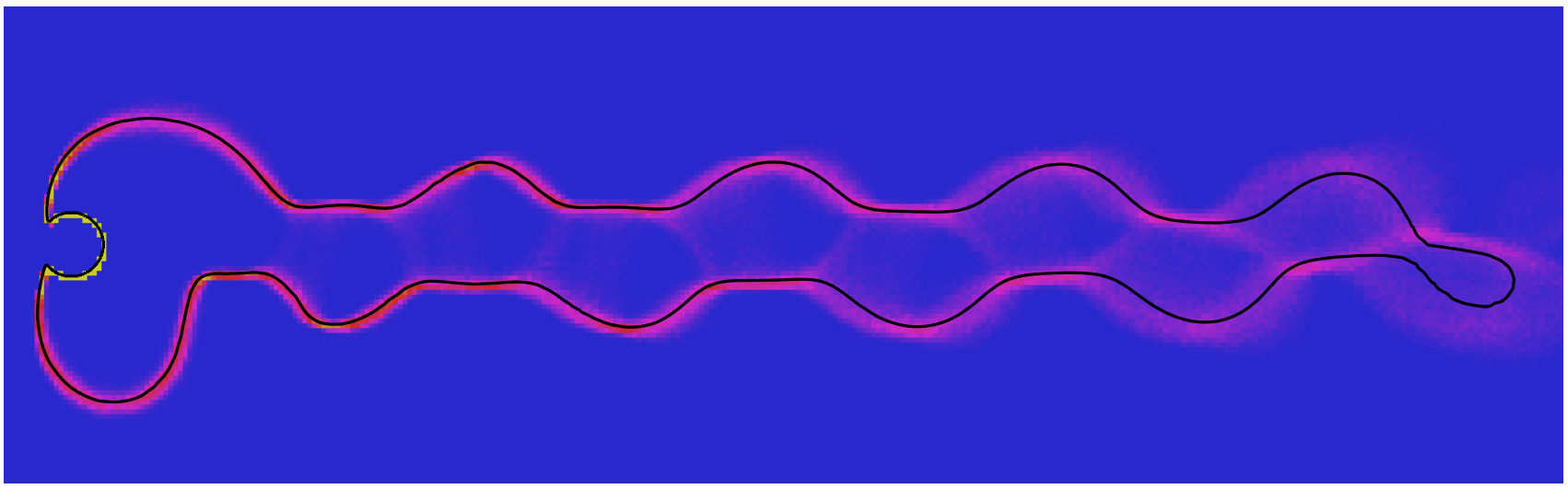

Fig. 7. (a) Ensemble of isocontours of the pressure field (b) Contour boxplot of the fluid flow simulation using 40 ensemble members with different perturbations in the initial conditions. (c) Demonstration of the isocontours of the mean and \pm 1 standard deviation of the pressure field. (d) Color mapped level set crossing probabilities of the pressure field calculated using the probabilistic marching cubes algorithm (with the mean pressure shown as a black contour line). The boxplots are rendered over the LIC visualization of the median member of the ensemble. 
the calibration pressure. Figure 7a shows the contours associated with this experiment. Figure. $7 \mathrm{~b}$ shows the result of using contour boxplots to visualize the variability of isocontours of the pressure field between different ensemble members at a specific timestamp (i.e., the captured cycles). As the $50 \%$ band suggests, the variability is mainly due to different lengths of the wake and different distances between eddies as they move down the eddy line. Unlike the output of the PMC algorithm, which is a continuous field of values, the contour boxplot is a set of quantitatively interpretable regions, and can be easily overlaid on top of other visualizations. Here we render the boxplots over a line integral convolution (LIC) flow visualization [3] of the median member of the ensemble for context. In practice, the specifics of the contour boxplot rendering would be adapted to the needs of a particular application.

Figures $7 \mathrm{c}-\mathrm{d}$ illustrate the comparison of our proposed method with alternative visualization schemes to convey the variability between ensemble members. Even though isocontours of sample mean and \pm 1 standard deviation of the pressure field (Fig. 7c) provide a quantitative description of the underlying variability between ensemble members, one can point out several problems with that approach. First, the mean is not representative of the population. The bulges associated with individual vortices/eddies are misshapen (flattened and elongated). Also, the separation of the vortices from this pressure contour at the end of the wake is lost. The variability is also misleading. The figure overestimates the variability perpendicular to the flow (up and down) and underestimates it along the flow (left and right). The contour boxplot captures these properties in a way that shows the proper shape of a typical wake and depicts the variability in a way that accurately represents the ensemble. Thus, this example demonstrates the isocontours extracted from mean and \pm 1 standard deviation of the pressure field do not satisfy the shape preserving property.

In recent years, there has been great interest in approximating and visualizing level-crossing probabilities [26, 28, 23, 27]. We have chosen to compare our method with the probabilistic marching cube (PMC) algorithm [28] as a representative of this collection of methods (here implemented in 2D) in Fig. 7d. In addition to the point-wise variability among ensemble members, PMC also takes into account the local spatial correlation of neighboring points. This algorithm uses a multivariate normal approximation for each neighborhood (estimated from the ensemble) and uses a Monte Carlo methods, with samples from this normal distribution, to empirically estimate the probability of an isocontour passing between 2 pixels (voxels, grid points). As in [28] we use $4 \times 4$ neighborhoods. These probabilities are then shown as an image with the color scheme proposed in [28]. Figure 7d shows the result of using PMC for the fluid simulation ensemble. In this rendering, blue denotes low probability values, while red and yellow are used to denote moderate and high probabilities, respectively. The mean suffers from problems similar to those in Figure 7c (they are slightly different because of differences in resolution). Furthermore, the variability, while quantitative and data driven, fails to capture essential aspects of the shapes in this ensemble and consistently underestimates the degree to which these individual eddies can vary in position forward and backward on the eddy line. Finally, both the field averaging and PMC methods are unable to systematically detect and convey the extreme behavior of several outliers.

In practice, such fluid simulations (as in real fluids) are only approximately cyclical. Low frequency variations (in time) will result in slower pulsations that can persist long after the initial transient effects of a simulation. Here we use contour boxplots to visualize the variability within a single simulation over time. For this example, the ensemble is the set of fields from a single simulation given by the sequence of minima in Fig. 6. Figure 8 shows the order statistics derived using contour boxplot for a window around the cylinder as an eddy forms and get disconnected. We can see that there is variability, but the distribution is geometrically symmetric and compact without any significant outliers

The last set of experiments uses the weather forecast data publicly available from the National Oceanic and Atmospheric Administration (NOAA) [1]. In climate research and weather forecast applications,

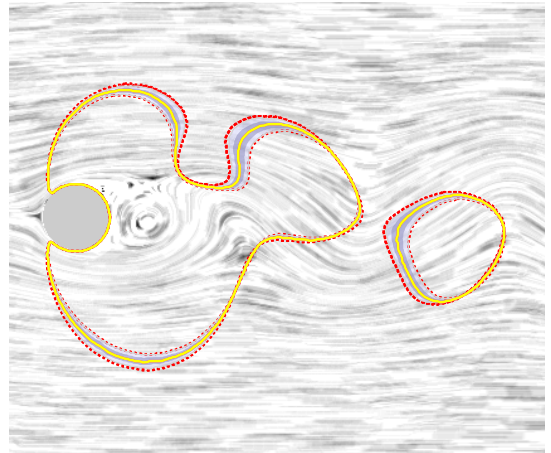

Fig. 8. Contour boxplot of a region of interest for the fluid flow simulation. In this figure, the trimmed mean is overlapping the median and hence is not visible in the figure.

ensemble data are formed through different runs of a simulation model with different perturbation of the initial conditions to account for the errors in the initial conditions and/or model parameterizations. Users are often extracting 2D slices of the atmosphere to study specific features such as temperature or humidity at different height levels to interpret weather predictions. For this experiment, we use isocontours of the temperature field at $500 \mathrm{mb}$ and a temperature $=-15 \mathrm{C}$ of SREFCONUS $(40 \mathrm{~km})$. Currently, one of the main tools available to domain experts to interpret weather forecast ensembles is the spaghetti plots [8] of isocontours provided by NOAA. The spaghetti plots are also available as animations through 87 forecast hours (with 3-hour periods). Using spaghetti plots, the variability is usually inferred as the extent to which the set of lines is spread out and how the patterns change through time. Using contour boxplots, one can quantitatively show the variability of the ensemble members. The dynamic version of the contour boxplot for this example (an animation) demonstrates also how the percentile bands evolve and expand (solutions diverge) as the simulation progresses over forecast time.

Figure. 9b shows the spaghetti plots provided by NOAA for the same forecast hour, and Fig. 9c shows a visualization of the isocontours of the averaged temperature field along with \pm 1 standard deviation. Finally, Fig. 9d demonstrates the level-crossing probabilities of temperature using the probabilistic marching cubes [28]. Figure 9 confirms that both of the pixel-based statistical methods lose a significant amount of detail and do not properly convey the extent to which even the most similar subset of samples varies. On the other hand, cBD not only preserves the details of the variability but also has the flexibility to provide quantitative information about the shapes and regions.

For all of these results, the cBD analysis was computed using MAT$\mathrm{LAB}$, and analysis times ranged from 10 to 20 minutes. This computation time is dominated by the large number of comparisons between different images to create bands. These computations are independent (and therefore easily parallelizable) at the level of images (coarse grain) and pixels (fine grain), and thus the method will run at interactive rates on state-of-the-art parallel architectures.

\section{SummaRY AND CONCLUSIONS}

This paper introduces contour boxplots for displaying statistical quantities from ensembles of isocontours or level sets associated with ensemble data. The method relies on the statistical notion of data depth, and for this we have introduced a new formulation of band depth, which is applicable to sets, generally, as well as isocontours. We have shown results comparing against other representative, quantitative methods that demonstrate that contour boxplots convey the quantitative and qualitative aspects of an ensemble of shapes, satisfying our design criteria in Section 1.

Limitations of the proposed method suggest some areas for future work. In some cases it may be important for the visualization to highlight more detail about the underlying distribution, such as the existence of multiple modes. This may require a generalization of the methodology to accommodate clustering [13] or mixture modeling. 
(a)

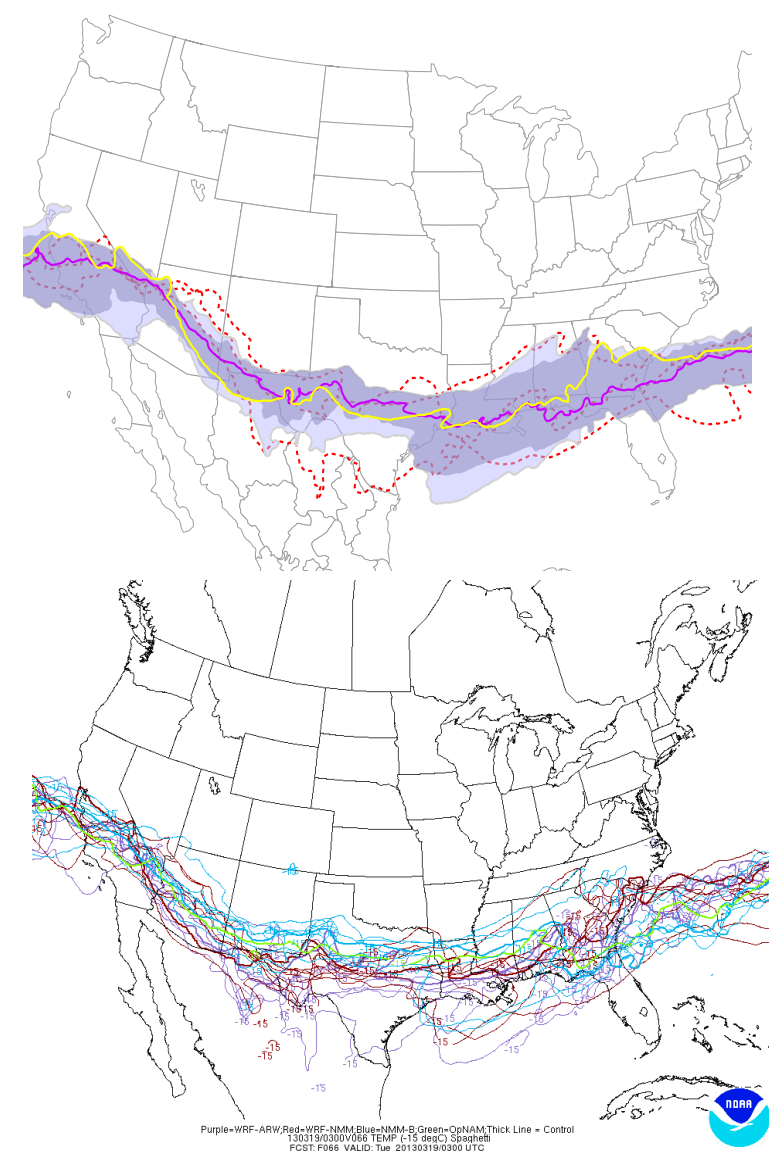

(c)

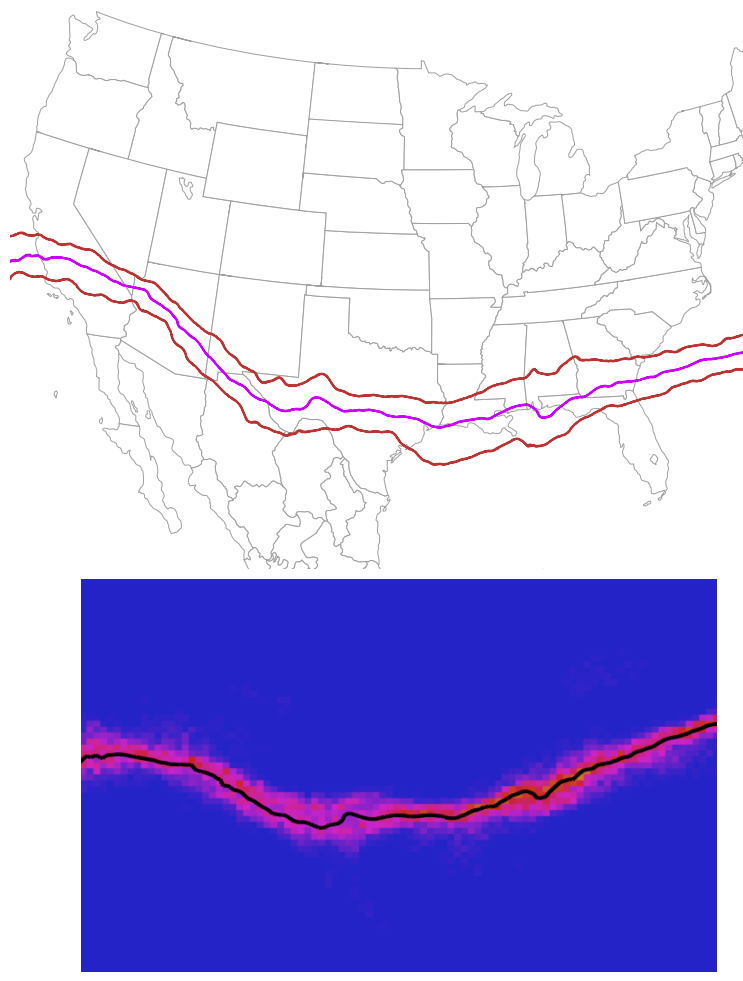

Fig. 9. (a) Contour boxplots of the temperature field at $500 \mathrm{mb}$ and temperature $=-15 \mathrm{C}$. (b) Spaghetti plots provided by NOAA. (c) Isocontours of the mean temperature field along with \pm 1 standard deviation. (d) Color mapped of the level-crossing probabilities of the temperature field calculated using the probabilistic marching cubes algorithm (with the mean temperature shown as a black contour line).
While the formulation generalizes to any dimension, we consider the $3 \mathrm{D}$ rendering problem as beyond the scope of this paper, but it is an important and interesting area for future work. Likewise, for timecritical, 3D applications, better computational strategies will become essential.

The proposed method is intended to be quite general, and thus we have demonstrated it in somewhat general contexts. Applications to specific problems might entail refinement of the rendering/display strategies including other backgrounds and contour rendering. Also under consideration is user input, in the form of querying specific instances or values and interactive control of parameters. Likewise, we have demonstrated a particular strategy for alignment of features in time that is specific to the fluid application, but more general strategies for such alignments are another important area of research.

The definition of set band depth and contour band depth is quite general and has a variety of applications in both the current context and beyond. For instance, there is a growing set of machine learning and statistical methods that rely on data depth and that are, in principle, now applicable to sets of contours. The basic properties of the set band depth and contour band depth warrant further investigation. Likewise, the methods in this paper open up other visualization possibilities, such as nonparametric methods for visualizing large collections of discrete sets or categorical data.

\section{A Appendix}

This Appendix provides the mathematical foundations of properties of the set and contour band depth formulations. The first proposition establishes that the proposed set band depth (sBD) is a generalization of function band depth as described in the literature [17].

Proposition 1: Set band depth (sBD) is equivalent to function band depth (BD) in the special case where SBD is applied to functions.

Proof sketch: Let $U=\mathscr{D} \times \mathscr{R}$ and let each element of $E$ correspond to the region of the graph below each function: $S_{i}=\left\{(x, y) \in \mathscr{D} \times \mathscr{R} \mid y \leq f_{i}(x)\right\}$. Given this, the union of a set of $S_{i} \mathrm{~s}$ gives the region in $U$ below the upper envelope of those functions and the intersection gives the region below the lower envelope. Another function $g$ lies in the band only if it is above the lower envelope and below the upper envelope, which is equivalent to the subset relationship given in Eq. 4.

In special cases, the application of $\mathrm{sBD}$ to contours is precisely the same as $\mathrm{BD}$ for functions. The following proposition shows the relation between $\mathrm{SBD}$ and functional band depth:

Proposition 2: For a collection of closed contours in the plane that is produced by an invertible transformation of an associated set of functions, sBD applied to the regions enclosed by those contours is equivalent to $\mathrm{BD}$ applied to the functions.

Proof sketch: Let $s U$ be the region in the plane that contains all of the contours. Let $\phi$ be the invertible map so that $\mathrm{s} U=\phi \circ U$. By Proposition 1, BD has an equivalent set representation on $U=\mathscr{D} \times \mathscr{R}$. The transformation $\phi$ commutes with these set operations (because it is invertible), and all of the probabilities are identical between the function and the contour representation. Figure $3 \mathrm{~b}$ shows a diagram of how this equivalence would be established.

Next we consider some specific properties of cBD. The first property shows that the method truly operates on closed contours and is independent of the definition of inside or outside.

Proposition 3: $\mathrm{cBD}$ is invariant with respect to sign changes on $F_{i}(x, y)$.

Proof sketch: Consider a cBD analysis on $F_{i}(x, y)=q$. If we consider a new analysis on $F_{i}^{\prime}(x, y)=-F_{i}(x, y)=q$, the associated sets $S_{i}^{\prime}$ on the new problem are the complements of their associated sets in the original problem, $S_{i}^{\prime}=S_{i}^{\mathrm{C}}$. If we substitute the $S_{i}^{\mathrm{C}}$ into Eq. 4 , we can commute it with the intersection operator (which becomes a union) and the union (operator), which becomes an intersection. We can then 
commute the complement with the subset operations, and they become superset operations, and the result is the sBD condition on the $S_{i}$-s and $F_{i}$-s. As pointed out above, care must be taken to define the subset operations on the interiors of sets.

Finally, we show that the band definition for contours captures a very important relevant property of the natural relationships between contours or level sets of functions.

Proposition 4: Consider the set band applied to isocontours of a collection of continuous, Morse functions $F_{i}$, where we test whether or not the $q$ contour of $F_{i}$ lies in the contour band associated with $F_{1}(x, y)=q, \ldots, F_{j}(x, y)=q$. Let $U$ be the contour associated with the union and $I$ be the contour associated with the intersection. Then $C=\left\{(x, y) \mid F_{i}(x, y)=q\right\}$ is in the contour set band if and only if there exists a (continuous, Morse) function $G(x, y)$ and $\varepsilon>0$ such that:

$$
\begin{array}{r}
I=\{(x, y) \mid G(x, y)=q+\varepsilon\}, \text { and } C=\{(x, y) \mid G(x, y)=q\}, \\
\text { and } U=\{(x, y) \mid G(x, y)=q-\varepsilon\} .
\end{array}
$$

Proof sketch: This proposition derives from basic properties of contour trees [4]. If a $G(x, y)$ exists to satisfy Eq. 10, then the sets $I_{R}=\{(x, y) \mid G(x, y)>q+\varepsilon\}, C_{R}=\{(x, y) \mid G(x, y)>q\}$, and $U_{R}=$ $\{(x, y) \mid G(x, y)>q-\varepsilon\}$ are nested, by construction, and satisfy the set band property. If the contours satisfy the set band property, then they are nested in a way that allows for the construction of a $G(x, y)$. If contours coincide, continuity in $G(x, y)$ would hold only in the limit as $\varepsilon \rightarrow 0$. Figure 4 shows some examples of how these nesting relationships can be satisfied or violated for different topologies of contours.

\section{ACKNOWLEDGMENTS}

This work was supported by National Science Foundation (NSF) grant IIS-1212806, IIS-0914564, and U.S. Department of Energy grant DEEE0004449.

\section{RefERENCES}

[1] National oceanic and atmospheric administration, 2012. http:// nomads. ncep. noaa.gov/.

[2] Nektar++, 2012. http: //www. nektar.info.

[3] B. Cabral and L. C. Leedom. Imaging vector fields using line integral convolution. In Proceedings of the 20th annual conference on Computer graphics and interactive techniques, SIGGRAPH '93, pages 263-270, New York, NY, USA, 1993. ACM.

[4] H. Carr, J. Snoeyink, and U. Axen. Computing contour trees in all dimensions. In Proceedings of the eleventh annual ACM-SIAM symposium on Discrete algorithms, pages 918-926, Philadelphia, PA, USA, 2000. Society for Industrial and Applied Mathematics.

[5] J. Cox, D. House, and M. Lindell. Visualizing uncertainty in predicted hurricane tracks. International Journal for Uncertainty Quantification, 3(2):143-156, 2013.

[6] S. Djurcilov, K. Kim, P. Lermusianax, and A. Pang. Visualizing scalar volumetric data with uncertainty. Computers and Graphics, 26:239-248, 2002.

[7] I. Dryden and K. Mardia. Statistical shape analysis. Wiley series in probability and statistics. Wiley, Chichester [u.a.], 1998.

[8] G. Fitzmaurice, M. Davidian, G. Verbeke, and G. Molenberghs. Longitudinal Data Analysis. Chapman \& Hall/CRC Handbooks of Modern Statistical Methods. Taylor \& Francis, 2008.

[9] G. Grigoryan and P. Rheingans. Probabilistic surfaces: Point based primitives to show surface uncertainty. In Proceedings of IEEE Visualization 2002, pages 147-153, 2002.

[10] G. Grigoryan and P. Rheingans. Point-based probabilistic surfaces to show surface uncertainty. IEEE Trans. Visualization and Computer Graphics, 10:564-573, 2004.

[11] M. Harris, A. AE, E. BJ, S. Maas, C. Peters, and J. Weiss. Finite element prediction of cartilage contact pressures in normal human hips. Journal of Orthopaedic Research, 30(7):1133-1139, 2012.

[12] C. Johnson and A. Sanderson. A next step: Visualizing errors and uncertainty. IEEE Computer Graphics and Applications, 23, 2003.

[13] R. Jornsten. Clustering and classification based on the 11 data depth. Journal of Multivariate Analysis, 90(1):67-89, 2004.

[14] A. J. Jospeh, S. K. Lodha, J. C. Renteria, and A. Pang. Uisurf: Visualizing uncertainty in isosurfaces. In Proceedings of the Computer Graphics and Imaging, pages 184-191, 1999.
[15] J. Kniss, R. Van Uitert, A. Stephens, G.-S. Li, T. Tasdizen, and C. Hansen. Statistically quantitative volume visualization. In Proceedings of IEEE Visualization 2005, pages 287-294, October 2005.

[16] S. Lodha, C. Wilson, and R. Sheehan. Listen: sounding uncertainty visualization. In Proceedings of IEEE Visualization 1996, pages 189-195, November 1996.

[17] S. Lopez-Pintado and J. Romo. On the concept of depth for functional data. Statistics and Econometrics Working Papers ws063012, Universidad Carlos III, Departamento de Estadstica y Econometra, May 2006.

[18] S. Lopez-Pintado and J. Romo. On the concept of depth for functional data. Journal of the American Statistical Association, 104(486):718-734, 2009.

[19] A. Pang, C. Wittenbrink, and S. Lodha. Approaches to uncertainty visualization. The Visual Computer, 13:370-390, 1997.

[20] A. T. Pang, C. M. Wittenbrink, and S. K. Lodh. Approaches to uncertainty visualization. The Visual Computer, 13:370-390, 1996.

[21] M. Pauly, N. Mitra, and L. Guibas. Uncertainty and variability in point cloud surface data. In Proc. Eurographics Symp. Point Based Graphics, pages 77-84, 2004.

[22] C. Petz, K. Pöthkow, and H.-C. Hege. Probabilistic local features in uncertain vector fields with spatial correlation. Computer Graphics Forum, 31:1045-1054, 2012.

[23] T. Pfaffelmoser, M. Reitinger, and R. Westermann. Visualizing the positional and geometrical variability of isosurfaces in uncertain scalar fields. Computer Graphics Forum, 30(3):951-960, 2011.

[24] T. Pfaffelmoser and R. Westermann. Visualization of global correlation structures in uncertain 2d scalar fields. In Computer Graphics Forum, volume 31, pages 1025-1034. Wiley Online Library, 2012.

[25] M. N. Phadke, L. Pinto, O. Alabi, J. Harter, R. M. Taylor II, X. Wu, H. Petersen, S. A. Bass, and C. G. Healey. Exploring ensemble visualization. pages 82940B-82940B-12, 2012.

[26] K. Pöthkow and H.-C. Hege. Positional uncertainty of isocontours: Condition analysis and probabilistic measures. Visualization and Computer Graphics, IEEE Transactions on, 17(10):1393 -1406, oct. 2011.

[27] K. Pöthkow, C. Petz, and H.-C. Hege. Approximate level-crossing probabilities for interactive visualization of uncertain isocontours. International Journal for Uncertainty Quantification, 3(2):101-117, 2013.

[28] K. Pöthkow, B. Weber, and H.-C. Hege. Probabilistic marching cubes. Computer Graphics Forum, 30(3):931-940, 2011.

[29] K. Potter, P. Rosen, and C. R. Johnson. From quantification to visualization: A taxonomy of uncertainty visualization approaches. In A. Dienstfrey and R. Boisvert, editors, Uncertainty Quantification in Scientific Computing, volume 377 of IFIP Advances in Information and Communication Technology, pages 226-249. Springer Berlin Heidelberg, 2012.

[30] K. Potter, A. Wilson, V. Pascucci, D. Williams, C. Doutriaux, P.-T. Bremer, and C. Johnson. Ensemble-vis: A framework for the statistical visualization of ensemble data. In Data Mining Workshops, 2009. ICDMW '09. IEEE International Conference on, pages 233-240, 2009.

[31] P. J. Rhodes, R. S. Laramee, R. D. Bergeron, and T. M. Sparr. Uncertainty visualization methods in isosurface volume rendering. In Eurographics 2003, Short Papers, pages 83-88, 2003.

[32] P. J. Rousseeuw, I. Ruts, and J. W. Tukey. The bagplot: A bivariate boxplot. The American Statistician, 53(4):382-287, November 1999.

[33] J. Sanyal, S. Zhang, J. Dyer, A. Mercer, P. Amburn, and R. Moorhead. Noodles: A tool for visualization of numerical weather model ensemble uncertainty. IEEE Transactions on Visualization and Computer Graphics, 16(6):1421-1430, 2010.

[34] D. Stephenson and F. Dolas-Reyes. Statistical methods for interpreting monte carlo ensemble forecasts. Tellus A, 52(3), 2011.

[35] Y. Sun and M. G. Genton. Functional boxplots for complex data visualization, 2009

[36] Y. Sun and M. G. Genton. Functional Boxplots. Journal of Computational and Graphical Statistics, 20(2):316-334, June 2011

[37] J. W. Tukey. Mathematics and the Picturing of Data. In International Congress of Mathematicians 1974, volume 2, pages 523-532, 1974.

[38] S. F. Wojtkiewicz, M. S. Eldred, R. V. Field, A. Urbina, and J. R. Redhorse. Uncertainty quantification in large computational engineering models. In In Proceedings of the 42rd AIAA/ASME/ASCE/AHS/ASC Structures, Structural Dynamics, and Materials Conference, number AIAA-2001-1455, 2001. 